\title{
Obesidad como factor de riesgo en pacientes con covid- 19: características clínicas e implicaciones. A propósito de un caso
}

Obesity as a risk factor in patients with covid-19: clinical characteristics and implications. About a case

\author{
Carlos Alberto Molina Cárdenas. ${ }^{1}$, Andreina Beatriz Borbor Cabrera. ${ }^{2}$, Ana Cecilia \\ Vinueza Mite. ${ }^{3} \&$ Carlos Adeodato Ricaurte Guerrero. ${ }^{4}$
}

\begin{abstract}
.
Introduction: Obesity is a chronic disease associated with increased morbidity and mortality worldwide, and the impact of the COVID-19 pandemic. The clinical manifestations of COVID-19 disease vary from asymptomatic to moderate and even severe cases, arriving with artificial airway and invasive ventilatory assistance to an Intensive Care
\end{abstract}

\section{Resumen.}

Introducción: La obesidad es una enfermedad crónica asociada a incremento de la morbimortalidad a nivel mundial, y el impacto con la pandemia de COVID-19. Las manifestaciones clínicas de la enfermedad por COVID-19 varían dese casos asintomáticos a moderados e incluso severos, llegando con vía aérea artificial y con asistencia ventilatoria

1 Licenciado en Terapia Respiratoria, Hospital de Especialidades Dr. Teodoro Maldonado Carbo, Guayaquil, Ecuador, albertocar190@hotmail.com, (iD) https://orcid.org/0000-0003-2677-6491

2 Médico General, Hospital de Especialidades Dr. Teodoro Maldonado Carbo, Guayaquil, Ecuador, andreina-borbor@hotmail.com, (i) https://orcid.org/0000-0003-2769-4308

${ }^{3}$ Licenciada en Enfermería, Universidad Estatal de Guayaquil, Facultad de Ciencias Médicas, Guayaquil, Ecuador, ana.vinuezam@ug.edu.ec, (iD) https://orcid.org/0000-0002-3272-0665

4 Médico General en Libre Ejercicio, Centro Médico Popular Vinces, Vinces, Ecuador, cricaurteg@unemi.edu.ec, (iD) https://orcid.org/0000-0003-2718-3620 
Unit (ICU). Methodology: A invasiva a una Unidad de Cuidados retrospective study was carried out, by collecting data from the electronic medical record of the AS 400 hospital system of the Hospital de Especialidades Dr. Teodoro Maldonado Carbo in January 2021. Results: 67-year-old obese female patient with a history of hypertension and diabetes milletus Type II, chest CT showed ground-glass infiltrates in both lung fields, multilobular with peripheral distribution, COVID-19 pneumonia. Contributions: Recognize possible mechanisms and implications in the clinical management of patients with morbid obesity, diagnosed with Pneumonia due to COVID 19.

Keywords: obesity, COVID-19, mortality, medicine, clinical case

Intensivos (UCI). Metodología: Se realizó un estudio retrospectivo, mediante recolección de datos de la historia clínica electrónica del sistema hospitalario AS 400 del Hospital de Especialidades Dr. Teodoro Maldonado Carbo enero 2021. Resultados: De los 9 artículos analizados en su totalidad, fueron publicadas en revistas internacionales, en el año 2020 y 2021, en relación con los resultados clínicos se evidencio una alta tasa de mortalidad en pacientes con obesidad que fueron diagnosticados con neumonía por COVID-19, con comportamiento de un estado proinflamatorio, produciendo de manera directa desregulación del sistema inmune. Aportes: Reconocer posibles mecanismos e implicaciones en el manejo clínico de pacientes con obesidad mórbida, con diagnóstico de Neumonía por COVID 19.

Palabras claves: obesidad, COVID-19, mortalidad, medicina, caso clínico.

\section{Introducción}

La enfermedad por coronavirus 2019 (COVID-19) desde su primera identificación en Wuhan, China en diciembre de 2019 es una enfermedad infectocontagiosa, con una propagación rápida, que si no se utilizan las medidas adecuadas de protección esta tendrá un rápido aumento de casos y muertes.

La presencia de neumotórax es habitual en el paciente que se encuentra con ventilación mecánica invasiva (VMI), sin embargo, ahora en el contexto de la actual pandemia por el SARS CoV2 hay reportes de casos de neumotórax espontaneo como una complicación rara de la enfermedad, sin antecedente de barotrauma inducido por presión positiva en las vías respiratorias (Pinos et al., 2021).

En una serie de 99 pacientes publicada por Yuan el neumotórax se presentó solo en el 12\% de los pacientes con COVID 19 (Yuan et al., 2021). 
Estudios reportan una mediana de tiempo de 24.3 días desde la admisión hospitalaria para el desarrollo de esta complicación en el caso de ser espontaneo, y cuando se presenta por barotrauma en promedio se desarrolla al 4-5 día post-intubación (Quincho-López et al., 2020).

Los hallazgos comunes de COVID-19 en los estudios de imagen tanto radiografía simple como tomografía de tórax están caracterizados por un patrón neumónico bilateral, con opacificación en vidrio deslustrado extenso, periférico, subpleural o posterior que envuelve los lóbulos inferiores principalmente; sin embargo, las características poco comunes pueden presentarse como efusión pericárdica o pleural, linfoadenopatía, cavitaciones, neumotórax y neumomediastino (Miranda et al., 2021).

Luego de la masificación en la utilización de la tomografía computarizada de tórax como herramienta principal en la identificación de casos tempranos de neumonía por COVID19, la detección de las complicaciones secundarias a esta afectación también fue en aumento, incluyendo los fenómenos más comunes trombóticos o inflamatorios en orden de la provisión a tiempo y adecuada de tratamiento dirigido a dichos eventos (López et al., 2020).

En pacientes con obesidad mórbida obesidad mórbida (Índice de Masa Corporal (IMC) $\geq 40 \mathrm{~kg} / \mathrm{m} 2$ ) se asoció de manera fuerte e independiente con la mortalidad en pacientes hospitalizados menores de 50 años en la Unidad de Cuidados Intensivos (UCI); la obesidad mórbida también se asoció con intubación y ventilación mecánica.

En la mayoría de los casos la presentación por COVID-19 se presenta como una enfermedad leve o asintomática, pero en los casos graves puede llegar complicar el cuadro clínico y posterior la muerte.

Las manifestaciones clínicas de la enfermedad por COVID-19 varían desde casos asintomáticos a moderados e incluso severos; alrededor de un $80 \%$ de los pacientes se recupera exitosamente, pero el restante sufre complicaciones, progresa a síndrome respiratorio agudo y en algunos casos puede llegar a la muerte (Tenorio-Mucha \& Hurtado-Roca, 2020).

La obesidad mórbida ha preocupado al personal sanitario debido que la relacionan un factor de riesgo para la morbilidad y la mortalidad prematura, teniendo consecuencias para la salud y la calidad de vida de las personas que la padecen. La obesidad se considera un factor de riesgo para más de 20 condiciones crónicas, en estas se encuentran la diabetes tipo 2, hipertensión, dislipidemia, enfermedades cardiovasculares, ictus, apnea de sueño y más de 12 tipos de cáncer (Petrova et al., 2020).

Desde una perspectiva cardiovascular, los ensayos y la evidencia genética demuestran de manera concluyente que la obesidad (y el exceso de tejido adiposo) está relacionada con comorbilidades como la hipertensión, diabetes, enfermedad coronaria, accidente cerebrovascular, fibrilación auricular, enfermedad renal e insuficiencia cardíaca, entidades que contribuyen a desenlaces adversos en infecciones respiratorias. La 
resistencia a la insulina y función reducida de las células beta en pacientes con obesidad limitan la capacidad de evocar un metabolismo apropiado en respuesta al desafío inmunológico, lo que lleva a disglucemias durante infecciones graves, lo que se relaciona también con peores desenlaces en sepsis severa (Internista et al., 2020).

Es de conocimiento que la obesidad se asocia a un estado proinflamatorio de bajo grado, con incremento de citocinas (TNF- interleucina-6) que genera una desregulación de la respuesta inmune innata y adaptativa. Esta condición inmunitaria, en las personas con obesidad, conlleva una mayor susceptibilidad a las infecciones, una respuesta deficiente al tratamiento con antivirales y menor eficacia de las vacunas (Rubio \& Lesmes, 2021).

El SARS-CoV-2 penetra en la célula empleando como receptor a la enzima convertidora de angiotensina 2 (ECA-2 por sus siglas en inglés), una exopeptidasa de membrana presente fundamentalmente en el riñón, los pulmones, corazón, páncreas, intestino delgado y endotelio vascular (Sanitarias, 2021). Tras contactar con el virus, se produce un fenómeno de down-regulation de la ECA-2, generando acumulación de angiotensina2 , que se cree que es la responsable del daño tisular pulmonar y del síndrome de distrés respiratorio, por sus propiedades vasoconstrictoras y fibróticas (Rubio \& Lesmes, 2021).

Un nuevo estudio, publicado por la Sociedad Española de Cirugía de la Obesidad ([SECO], 2021), según el cual:

“aún no está claro si este es el resultado de una mayor expresión de ACE-2 en los adipocitos de personas con obesidad o que tienen más tejido adiposo en general (y, por lo tanto, un mayor número de células que expresan ACE-2). Por ende, no se descarta la posibilidad de que la grasa intratorácica (pulmón), epicárdica (corazón), perirrenal (riñón) y mesentérica (intestino) del tejido adiposo de las personas con obesidad puede ser un objetivo potencial y un reservorio viral y diseminación para el SARS-CoV-2 antes de que se propague a otros órganos, como ha demostrado ser el caso de otros virus".

La obesidad también se acompaña de complicaciones médicas (HTA, diabetes tipo 2, enfermedades cardiovasculares), un estado de hipercoagulabilidad y trombosis, que determinan un peor pronóstico frente a la infección por COVID-19. Asimismo, las personas con obesidad presen- tan mayores dificultades respiratorias por resistencia al flujo aéreo, menor expansión del volumen pulmonar, dificultades de movilización de la caja torácica (diafragma y músculos intercostales), que van a ser responsables de hipoventilación, hipertensión pulmonar y apneas del sueño (Rubio \& Lesmes, 2021). Las observaciones clínicas apuntan a que, cuando la respuesta inmune no es capaz de controlar eficazmente el virus, como en personas mayores con un sistema inmune debilitado, el virus se propagaría de forma más eficaz produciendo daño tisular pulmonar, lo que activaría a los macrófagos y granulocitos y conduciría a la liberación masiva de citoquinas pro-inflamatorias (Sanitarias, 2021). Esta rápida reacción en cadena causada por el SLC usualmente genera en forma apresurada, lesiones inflamatorias en múltiples órganos y falla aguda funcional. El fenómeno de SLC relacionado con infección por COVID-19, está caracterizado por: 1) disminución de circulación de linfocitos T CD4, 
CD8 y células NK, 2) incremento de IL-6 en sangre periférica, 3) fiebre continua, 4) daño de tejidos y órgano causado por reacción inmune debido a liberación de citoquinas y 5) disfunción en coagulación (Ame et al., 2020).

La obesidad por si sola favorece la trombosis, una de las complicaciones frecuentes reportadas en series de casos de análisis anatomopatológicos de pacientes fallecidos por COVID-19 severo, estableciendo una asociación entre COVID-19 severo, coagulación intravascular diseminada protrombótica y altas tasas de tromboembolismo venoso, favorecida por la obesidad (Ángeles et al., 2021). Se ha observado que los niveles de antitrombina son menores en casos de COVID-19, y los niveles de dímero D y fibrinógeno son mayores que en población general. Además, la progresión de la gravedad de la enfermedad va ligada a un aumento gradual del dímero D. Estos hallazgos apoyan la teoría del desarrollo de una coagulopatía de consumo en infecciones por SARS-CoV2, y que cuando estas ocurren empeora el pronóstico (Sanitarias, 2021).

Los estudios muestran que la obesidad es un factor de riesgo para la hospitalización el ingreso en UCI, y el desarrollo de consecuencias graves, debido a que durante la estancia hospitalaria de pacientes con obesidad mórbida con diagnostico por COVID-19 se han podido observar una alta mortalidad, debido a que durante el ingreso hospitalario puede sobrevenir complicaciones, como son las coinfecciones bacterianas y fúngicas, y neumonías asociadas a la ventilación mecánica en aquellos pacientes con vía aérea artificial y asistidas a ventilación mecánica invasiva.

\section{Metodología}

El estudio y comprensión de los problemas humanos exige una amplia pluralidad metodológica, seleccionar uno u otro método a utilizar, dependerá del problema concreto a evaluar (Palella y Martins, 2012, p. 42).

La justificación del problema, indica la razón por la cual se quiere realizar dicha investigación, y el aporte que se pretende lograr en los distintos ámbitos, sean estos: sociales, políticos, económicos, humanos, etc. (Palella y Martins, 2012, p. 60).

Al definir los objetivos se determina qué tipo de investigación se realizará y deberá establecerse: el diseño, tipo, nivel y modalidad de estudio (Palella y Martins, 2012, p. 84).

La presente investigación aplica el método cuantitativo, diseño bibliográfico, tipo documental, de corte transversal, analítico explicativo con los objetivos del estudio por el método de causa efecto, sin intervención del investigador.

Se realizó un estudio retrospectivo, mediante recolección de datos de la historia clínica electrónica del sistema hospitalario AS 400 del Hospital de Especialidades Dr. Teodoro Maldonado Carbo enero 2021, revisión de literatura en buscadores Uptodate, PubMed, Clinicalkey. 


\section{Caso Clínico}

Se trata de una paciente femenina obesa de 67 años de edad con antecedentes importantes de hipertensión arterial de larga data controlada con enalapril y diabetes mellitus tipo 2 controlada con insulina de acción rápida 10 UI en la mañana y 5 UI en la noche, quien presento un cuadro clínico de 7 días de evolución aproximadamente, que se caracterizó por presentar tos poco productiva, malestar general sensación de alza térmica y dificultad respiratoria, por lo cual acude al servicio de triaje respiratorio del Hospital de Especialidades Dr. Teodoro Maldonado Carbo, se le realizo valoración clínica, química sanguínea, exámenes por imágenes, en la TAC de tórax inicial se observó (fig.1) infiltrados en vidrio deslustrado en ambos campos pulmonares, multilobular, de distribución periférica, prueba para SARS-Cov2 con resultado positivo por lo cual fue ingresada al área de emergencia con interconsulta a la Unidad de Cuidados Intensivos (UCI).

\section{Figura 1}

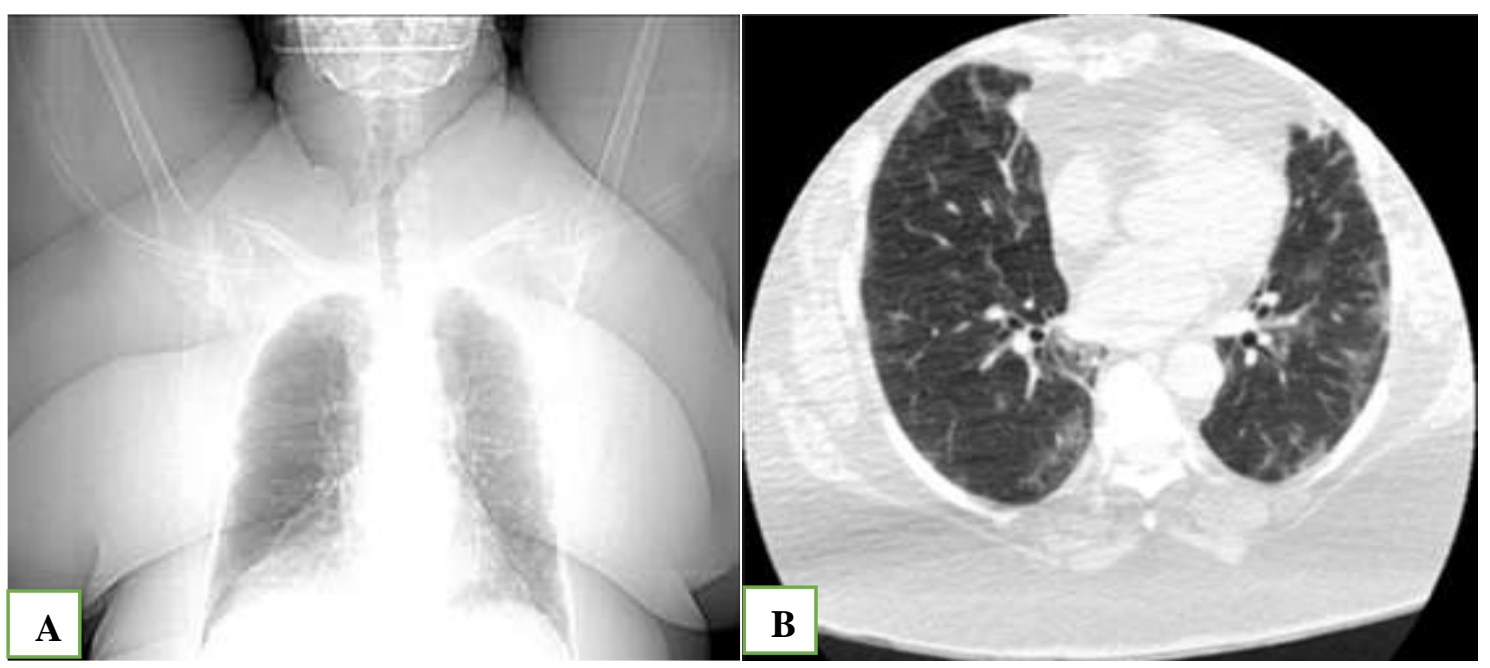

A. Paciente con obesidad mórbida, con diagnostico por COVID-19. B. Infiltrados en vidrio deslustrado, neumonía por COVID-19.

\section{Evolución}

Paciente en emergencia con manejo integral para COVID-19 sin evidencia de mejoría clínica, permanece taquipneica no tolera el decúbito, ha realizado hiperglucemias corregidas con insulina rápida, a la auscultación murmullo vesicular disminuido globalmente, aislados estertores crepitantes bibasales, FR 25 RPM; en la TAC de tórax inicial se observó (fig.1) infiltrados en vidrio deslustrado en ambos campos pulmonares, multilobular, de distribución periférica.

Tras permanecer 4 horas en el área de emergencia se la derivó a la UCI con diagnóstico de neumonía viral por COVID-19, respirando por cánula de alto flujo, con flujo 50 1/min, FIO2. 90\% alcanzando Sat.87\%; siendo valorada nuevamente y confirmando su diagnóstico por COVID-19 se decide tratamiento a base de corticoides y antibióticos; sin embargo, en el transcurso de las horas la paciente presenta empeoramiento de su cuadro 
clínico, presentando mayor dificultad respiratoria con aumento de los requerimientos de oxígeno y aumento de la fracción inspiratoria de oxígeno (FIO2).

En las últimas 48 horas de ingreso a UCI sin mejoría clínica, al examen físico se puede observar apariencia general regular, a nivel del tórax elasticidad y expansibilidad disminuida, tiraje subcostal, taquipnea, murmullo vesicular disminuido en ambos campos pulmonares, sin evidencia de mejoría en los controles gasométricos, obteniendo PH: 7.44, PO2: 52 mmHg, PCO2: $38.1 \mathrm{mmHg}$, HCO3: $25.5 \mathrm{mEq} / \mathrm{L}, \mathrm{BE}: 1.4 \mathrm{mEq} / \mathrm{L}$, PAFIO2: 68 $\mathrm{mmHg}$, posteriormente se le da tratamiento de VMNI modalidad BPAP (FIO2: 85\%, TI: 1.0, PEEP: 10 cmH2O, PS: 14, FR. 12 r/min obteniendo saturación de 85\%, en exámenes de laboratorio se evidencia leucocitosis más neutrofilia y linfopenia, elevación de reactantes de fase aguda, elevación de azoados, catalogándose como una injuria renal aguda grado II, ferritina y dinero D por encima de valores referenciales, en examen radiológico se evidencian infiltrados de predominio periférico compatibles con enfermedad por COVID-19, por lo que se decide rotación de antibiótico por posible sobreinfección, además de terapia con corticoides inmunomoduladores y anticoagulación.

Posterior al tratamiento no invasivo se decide sedoanalgesia e intubación orotraqueal, tubo Shiley No 8, y asistencia ventilatoria invasiva modalidad IPPV: FIO2. 100\%, VT: 300 ml, TI: 1.00, FR: 20 r/min, PEEP: 14 cmH2O, Flujo: 0.60. no se evidencio mejoría en los controles gasométricos, $\mathrm{PH}: 7.31$, PO2: $79 \mathrm{mmHg}, \mathrm{PCO} 2: 45 \mathrm{mmHg}, \mathrm{HCO}$ : 21.1 $\mathrm{mEq} / \mathrm{L}, \mathrm{BE}$ : $3.9 \mathrm{mEq} / \mathrm{L}$, Sat. $96.1 \%$, PAFIO2: $79 \mathrm{mmHg}$. Se le realizo interconsulta a cirugía general para realizarle traqueotomía temprana.

Paciente en decúbito prono con 96 horas de hospitalización en UCI con intubación orotraqueal, bajo efecto de sedoanalgesia y vasopresores, con asistencia ventilatoria invasiva con parámetros de protección pulmonar modalidad IPPV, antibioticoterapia y uso de corticoides; sin clara evidencia de mejoría clínica, con inestabilidad hemodinámica, sin evidencia de mejoría en gasometría arterial con hipoxemia, PAFIO2: $75 \mathrm{mmHg}$, catalogándose como SDRA grave, además sin clara evidencia de mejoría en exámenes de laboratorio con predominio de leucocitosis, neutrofilia y linfopenia.

Fallece tras 120 horas de hospitalización en la Unidad de Cuidados Intensivos (UCI).

\section{Resultados}

De los 9 artículos analizados en su totalidad, fueron publicadas en revistas internacionales, en el año 2020 y 2021, en relación con los resultados clínicos se evidencio una alta tasa de mortalidad en pacientes con obesidad que fueron diagnosticados con neumonía por COVID-19, con comportamiento de un estado proinflamatorio, produciendo de manera directa desregulación del sistema inmune. En casos leves al momento del ingreso los pacientes requirieron oxigenoterapia mediante dispositivos de bajo y alto flujo, como, por ejemplo, cánula nasal, mascarilla simple de oxígeno y cánula de alto flujo; y en los casos más graves se requirió el uso de ventilación mecánica no invasiva y/o la utilización de una vía aérea artificial con asistencia ventilatoria mecánica invasiva con prolongación de la estancia hospitalaria. Teniendo a 
la obesidad como posible factor que aumenta la mortalidad en pacientes que han sido diagnosticado por infección por COVID-19.

\section{Discusión}

El coronavirus es un virus de etiología zoonótica que hasta la el 28 de noviembre de 2021, alrededor de 5,21 millones de personas habían fallecido a nivel mundial a consecuencia de la COVID-19. Mientras que, en Asia, continente en el que se originó el brote, la cifra de muertes ascendía a alrededor de un millón de personas, los decesos en Europa superan en más de 400.000 personas dicha cifra. En concreto, se han registrado aproximadamente un millón y medio de muertes por el coronavirus en el Viejo Continente. Sin embargo, ya no es el continente con mayor número de fallecidos por COVID-19. La cifra contabilizada en América superaba ya los 2,3 millones de decesos ese día.

Por otra parte, se considera como diagnóstico antropométrico de obesidad un (índice de masa corporal) IMC igual o mayor a $30 \mathrm{Kg} / \mathrm{m} 2$ para todas las poblaciones del mundo con excepción de China, quienes toman como punto de corte de $>27,5 \mathrm{Kg} / \mathrm{m} 2$ (Tartof et al., 2020).

Se presenta el caso de una paciente con obesidad mórbida diagnosticada con neumonía por COVID-19 con fracaso de tratamiento VMNI y posterior entubación endotraqueal por descompensación cardiorrespiratoria, frecuentemente se manifiesta como una infección respiratoria aguda de respuesta leve con síntomas como fiebre, tos y dificultad respiratoria que en la mayoría de la población no progresa. No obstante, en algunos individuos puede progresar a una infección respiratoria severa, con necesidad de unidad de cuidados intensivos (UCI) y ventilación mecánica invasiva.

En la infección por SARS-CoV-19 se ha observado una respuesta exagerada caracterizada principalmente por secreción de citocinas proinflamatorias aberrantes, asociada a gran cantidad de macrófagos alveolares, con disminución óxido de células T-CD4 + y T-CD8 +, pero no en células B, con sobreproducción de IL-6, IL-2R, IL-10 y TNF $\alpha$, disminución de la expresión de IFN $\gamma$ lo que se correlacionó con la gravedad de la enfermedad (Ame et al., 2020).

Además de los efectos deletéreos sobre la inmunidad del huésped, en el contexto de la enfermedad por el nuevo coronavirus, se ha demostrado que la obesidad afecta la función pulmonar de varias formas, relacionadas con el aspecto mecánico e inflamatorio, aumento de la expresión de ACE2 (angiotensina-convertingenzima 2), mayor diversidad y títulos virales y eliminación prolongada del virus, reducción del volumen espiratorio y capacidad vital forzada. Así, hace que la persona con obesidad sea más susceptible a presentar síntomas respiratorios y favorecer la progresión a insuficiencia respiratoria (João et al., 2021).

Por último, los pacientes con obesidad en la atención sanitaria deben ser considerados y tratados como una población de alto riesgo para el Covid-19, en virtud de lo cual, es 
importante intensificar las medidas preventivas de contagio, así como la asistencia sanitaria especializada, en casos confirmados de Covid-19 (Ángeles et al., 2021).

\section{Conclusiones}

- Se pretende reconocer posibles mecanismos e implicaciones en el manejo clínico de pacientes con obesidad mórbida, con diagnóstico de Neumonía por COVID19. Además, es necesario crear una cultura que promueva los estilos de vida saludables y prevención de la obesidad.

- Es importante concienciar a la población en general sobre la severidad de la enfermedad por COVID-19, sobretodo en pacientes con obesidad que se ha constituido un factor de riesgo para mortalidad, razón por la cual debería ser tratadas como una población de alto riesgo, de manera que se intensifique las medidas de prevención contra contagio antes de la infección, proveyendo de manera integral asistencia especializada en aquellos pacientes que han sido diagnosticado neumonía por COVID-19. La presencia de obesidad no solo ha tenido impacto en cuanto a la presentación de manifestaciones clínicas de gran severidad hasta llegar a un síndrome de distrés respiratorio agudo con necesidad de ingreso a UCI, requerimiento de vía área artificial, asistencia ventilatoria invasiva, sino también es un factor de riesgo para la mortalidad.

\section{Referencias Bibliográficas}

Ame, L., Cl, I., Barranquilla, N., Lulle, C.A. \& Sabana, L. (2020). 29 2020. 29:10-14.

Ángeles, M.D.L., Wiesner, S. \& Katiuska, T. (2021). Morbi-Mortalidad en pacientes obesos infectados por COVID-19 Morbimortality in obese patients infected with COVID-19 Morbi-Mortalidade em pacientes obesos infectados com COVID-19. (2):71-79. doi:10.26820/recimundo/5.(2).abril.2021.71-79

Internista, M., Clínica, F., Departamento, D. \& Interna, D.M. (2020). RAEM. Published online. 58-64.

João, F., Neto, D.C. \& Loiola, B.M. (2021). Resultados y características clínicas de personas con obesidad y covid-19: Revisión integrativa. Published online 544-556.

López Vega, J. M., Parra Gordo, M. L., Diez Tascón, A., \& Ossaba Vélez, S. (2020). Pneumomediastinum and spontaneous pneumothorax as an extrapulmonary complication of COVID-19 disease. Emergency Radiology, 27(6), 727-730. https://doi.org/10.1007/s10140-020-01806-0

Miranda Solís, E. M., Ayala López, J. A., Heredia Calvopiña, P. A., \& Morales Cumbajín, R. E. (2021). Neumotórax espontáneo secundario a bullas enfisematosas como secuela de Covid-19 severo: reporte de caso. Anatomía Digital, 4(4), 129-140. https://doi.org/10.33262/anatomiadigital.v4i4.1938 
Palella, Santa. \& Martins, Feliberto. (2012). Metodología de la investigación cuantitativa (1ra reimpresión). Caracas: Fedupel ISBN: 980-273-445-4.

Petrova, D., Salamanca-Fernández, E., Rodríguez Barranco, M., Navarro Pérez, P., Jiménez Moleón, J.J. \& Sánchez, M.J. (2020). Obesity as a risk factor in COVID19: Possible mechanisms and implications. Aten Primaria. 52(7):496-500. doi: 10.1016/j.aprim.2020.05.003

Pinos Cedeño, M. J., Aguiar Flores, G. E., Layedra Ajila, M. E., \& Adriano Pérez, G. I. (2021). Neumotórax espontáneo y secundario a barotrauma en COVID 19, serie de casos y revisión de la literatura. Anatomía Digital, 4(4), 23-34. https://doi.org/10.33262/anatomiadigital.v4i4.1888

Quincho-Lopez, A., Quincho-Lopez, D. L., \& Hurtado-Medina, F. D. (2020). Case Report: Pneumothorax and Pneumomediastinum as Uncommon Complications of COVID-19 Pneumonia. American Journal of Tropical Medicine and Hygiene, 103(3), 1170-1176. https://doi.org/10.4269/ajtmh.20-0815

Rubio, M.A. \& Lesmes, B. (2021). Endocrinología, Diabetes y Nutrición Obesidad en tiempos de COVID-19. Un desafío de salud global. Endocrinol Diabetes y Nutr. 68(2):123-129. doi: 10.1016/j.endinu.2020.10.001

Sanitarias, E. (2021). Información Científica-Técnica Índice. Published

Sociedad española de cirugía de la obesidad [SECO]. (2021). Obesidad y COVID-19: por qué las personas con sobrepeso tienen más riesgo de enfermar gravemente. https://www.seco.org/Obesidad-y-COVID19-por-que-las-personas-consobrepeso-tienen-mas-riesgo-de-enfermar-gravemente_es_1_157.html

Tartof, S. Y., Qian, L., Hong, V., Wei, R., Nadjafi, R. F., Fischer, H., ... \& Murali, S. B. (2020). Obesity and mortality among patients diagnosed with COVID-19: results from an integrated health care organization. Annals of internal medicine, 173(10), 773-781. doi:10.7326/M20-3742

Tenorio-Mucha, J. \& Hurtado-Roca, Y. (2020). Revisión sobre obesidad como factor de riesgo para mortalidad por COVID-19. Acta Medica Perú. 37(3):324-329. doi:10.35663/amp.2020.373.1197

Yuan, X., Shanqing, L., \& Hongsheng, L. (2021). Clinical outcomes of pleural drainageon pneumothorax and hydrothorax in critically ill patients with COVID19: A case series with literature review. Heart and Lung, 50(2),213-219. https://doi.org/10.1016/j.hrtlng.2020.12.007

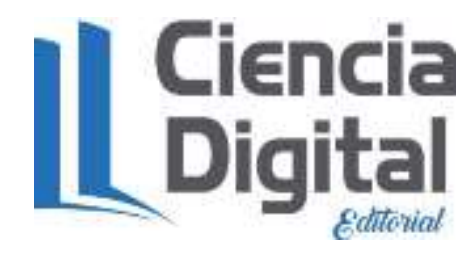




\section{PARA CITAR EL ARTÍCULO INDEXADO.}

Molina Cárdenas, C. A., Borbor Cabrera, A. B., Vinueza Mite, A. C., \& Ricaurte Guerrero, C. A. (2021). Obesidad como factor de riesgo en pacientes con covid-19: características clínicas e implicaciones. a propósito de un caso. Anatomía Digital, 4(4), 167-177. https://doi.org/10.33262/anatomiadigital.v4i4.1950

\section{LCiencia}

El artículo que se publica es de exclusiva responsabilidad de los autores y no necesariamente reflejan el pensamiento de la Revista Anatomía Digital.

El artículo queda en propiedad de la revista y, por tanto, su publicación parcial y/o total en otro medio tiene que ser autorizado por el director de la Revista Anatomía Digital.
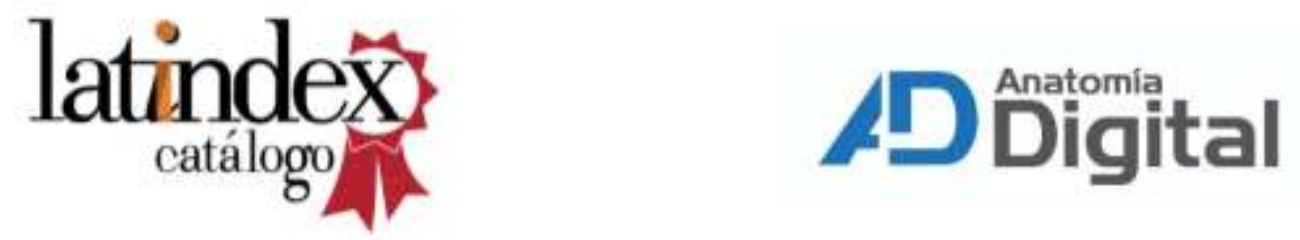